\title{
Aspectos da ecologia e do comportamento de flebotomíneos em área endêmica de leishmaniose visceral, Minas Gerais
}

\author{
Aspects of the ecology and behaviour of phlebotomines in endemic area for \\ visceral leishmaniasis in State of Minas Gerais
}

\author{
Ricardo Andrade Barata ${ }^{1}$, João Carlos França-Silva ${ }^{2}$, Wilson Mayrink ${ }^{2}$, Jaime Costa da \\ Silva $^{3}$, Aluízio Prata ${ }^{4}$, Elias Seixas Lorosa ${ }^{5}$, Jaqueline Araújo Fiúza ${ }^{1}$, Caroline Macedo \\ Gonçalves $^{1}$, Kênia Maria de Paula ${ }^{1}$ e Edelberto Santos Dias ${ }^{1}$
}

\begin{abstract}
RESUM0
O comportamento e hábitos alimentares de algumas espécies da flebotomíneos têm sido útil na compreensão da epidemiologia das leishmanioses. № município de Porteirinha (MG) , foram realizadas capturas mensais sistematizadas utilizando-se 28 armadilhas luminosas tipo CDC, durante o período de janeiro a dezembro de 2002. Foram capturadas 14 espécies de flebotomíneos, totalizando 1.408 exemplares. De acordo com o ambiente, os resultados obtidos mostraram que o peridomicílio apresentou a maior $(53,3 \%)$ porcentagem dos espécimens encontrados na região, embora parte (46,7\%) da fauna também tenha sido encontrada no intradomicílio. 0 repasto sanguíneo de 38 fêmeas de Lutzomyia longipalpis, provenientes do campo, foi identificado através da reação de precipitina. Os resultados indicam que Lutzomyia longipalpis foi a espécie predominante $(65,1 \%)$, mostrando-se oportunista, podendo sugar uma ampla variedade de vertebrados.
\end{abstract}

Palavras-chaves: Leishmaniose visceral. Lutzomyia. Ecologia de vetores. Preferência alimentar. Precipitina.

\begin{abstract}
Studies on the behavioral and feeding habits of some species of phlebotominae sand flies have contributed to the comprehension of the epidemiology of leishmaniasis. In the present work, systematic captures were performed monthly in the municipality of Porteirinha (MG) using 28 light traps (CDC) from January to December 2002. Fourteen different species of phlebotomine were captured in a total of 1,408 specimens. The highest percentage of individuals (53.3\%) was collected in the peridomicile against $46.7 \%$ in the intradomicile. Lutzomyia longipalpis was the predominant species in that region. The blood feeding of 38 females of this species from the field was analyzed by precipitin reaction. The results indicated that Lutzomyia longipalpis is an opportunist (65.1\%) species that feeds on a wide variety of vertebrates in nature.
\end{abstract}

Key-words: Visceral leishmaniasis. Lutzomyia. Vector ecology. Host preference. Precipitin.

Aleishmaniose visceral (IV) tem aumentado significativamente sua importância no contexto da saúde pública devido ao processo de urbanização e em decorrência das alterações no ambiente natural $^{25} 3436$. Algumas espécies de flebotomíneos que até então apresentavam comportamento silvestre têm sido encontradas perto de habitações humanas, em plantações de bananeiras e também em áreas florestais demonstrando que se encontram em processo de adaptação às modificações provocadas pelo homem ${ }^{27}$.

№ ciclo de vida da Leishmania chagasi (Cunha \& Chagas, 1937), agente etiológico da IV no Novo Mundo, a transmissão ocorre, principalmente, através da picada de fêmeas de flebotomíneos da espécie Lutzomyia longipalpis ( Lutz \& Neiva, 1912) ${ }^{1017}$. Esta espécie tem demonstrado uma grande capacidade

1. Centro de Pesquisas René Rachou da Fundação Oswaldo Cruz, Belo Horizonte, MG. 2. Universidade Federal de Minas Gerais, Belo Horizonte, MG. 3. Fundação Nacional de Saúde, Belo Horizonte, MG. 4. Faculdade de Medicina do Triângulo Mineiro, Uberaba, MG. 5. Instituto Oswaldo Cruz da Fundação Oswaldo Cruz, Rio de Janeiro, RJ.

Endereço para correspondência: Dr. Edelberto Santos Dias. Laboratório de Leishmanioses/CPqRR. Av. Augusto de Lima 1715, Barro Preto, 30190-002 Belo Horizonte, MG.

Fax 5531 3295-3566 ramal 158

e-mail: edel@cpqrr.fiocruz.br

Recebido para publicação em 8/11/2004

Aceito em 3/5/2005 
de se adaptar em vários ambientes, aumentando muito a densidade destes insetos dentro e ao redor de habitações humanas facilitando a transmissão da doença ${ }^{9}{ }^{20}$.

Em localidades onde a LV é endêmica, o cão (Canis familiaris) , como hospedeiro doméstico, tem sido incriminado como 0 principal reservatório de L chagasi no ciclo de transmissão para 0 homem nos centros urbanos s $^{6}{ }^{14}$. Outros animais foram descritos como reservatórios de leishmânias. Deane \& Deane ( 1954) relataram o primeiro registro de infecção em canídeos silvestres no continente americano, sendo a raposa Dusicyon vetulus, o reservatório incriminado ${ }^{6}$. Sherlock cols ${ }^{33}$ encontraram no Estado da Bahia, o Didelphis albiventris naturalmente infectado $0^{33}$. Este foi o primeiro registro no Novo Mundo de um reservatório silvestre marsupial para L chagasi. № Velho Mundo, como hospedeiros silvestres, têm sido descritos ainda 0 chacal, Canis aureus, 0 lobo, Canis lupus, e a raposa, Vulpes vulpes, encontrados em áreas rurais remotas ${ }^{23}$.

Contudo, L longipalpis tem sido observada alimentando-se de uma grande variedade de vertebrados, incluindo bois, cavalos, macacos, porcos e galinhas 2021223239 . Para definir a variação de hospedeiros e as preferências alimentares de L longipalpis sob condições naturais, nós realizamos a reação de precipitina com fêmeas capturadas em áreas urbanas do município de Porteirinha

0 conhecimento da fauna flebotomínica e 0 estudo do comportamento de algumas espécies têm sido objeto de vários investigações ${ }^{3} 4037$. A biologia do flebotomíneo nos ajuda a entender como cada espécie interage com seu habitate como a transmissão de leishmaniose pode estar ocorrendo em determinada área.

0 objetivo do presente estudo foi investigar a fauna flebotomínica, aspectos de comportamento das diferentes espécies capturadas, particularmente L. longipalpis, sua preferência alimentar e associar seu possível papel na transmissão da leishmaniose visceral no município de Porteirinha.

\section{MATERIAL E MÉTODOS}

Área de estudo. 0 município de Porteirinha localiza-se na região norte do Estado de Minas Gerais, zona do Alto Médio São Francisco, a $640 \mathrm{~km}$ de Belo Horizonte. Ocupa uma extensão de $1.769,59 \mathrm{~km}^{2}$, estando situado na área do "Polígono das Secas" entre 15 44' 42" - latitude sul, $43^{\circ} 01^{\prime} 46^{\prime \prime}$ - longitude oeste e altitude de $567 \mathrm{~m}$.

0 clima é do tipo tropical semi-úmido, quase sempre quente e com estação seca prolongada. As chuvas ocorrem entre os meses de outubro e março, registrando índices pluviométricos médios anuais de $600 \mathrm{~mm}$. A média anual de temperatura é de $27^{\circ} \mathrm{C}^{18}$.

0 relevo da região é bastante distinto, 0 que possibilita dividir a cidade em uma área elevada, de morros calcários, e uma mais baixa e plana representada pelo domínio da depressão São Franciscana.
A vegetação dominante é o cerrado, aparecendo ainda vegetações arbóreas mais densas nas partes úmidas dos vales, especialmente às margens dos rios pertencentes à Bacia do rio São Francisco.

A cidade possui 37.890 habitantes, sendo destes 18.140 residentes na área urbana e 19.750 na área rural ${ }^{19}$. As residências, de um modo geral, não possuem saneamento básico adequado, onde também é marcante a presença de animais como galinhas, porcos, cavalos e cães no intra e peridomicílio.

Métodos de captura. As capturas sistemáticas foram realizadas com armadilhas luminosas do tipo CDC $^{35}$ durante 0 período de janeiro a dezembro de 2002 . 0 município foi dividido em 7 bairros, tendo dois pontos por bairro, sendo que em cada ponto foi utilizada uma armadilha no intra e outra no peridomicílio pareadamente, levando-se em consideração a ocorrência de casos humanos e/ou a presença de cães com sorologia positiva para calazar. As armadilhas foram expostas das 18:00h às 8:00h da manhã seguinte, durante 5 dias consecutivos em cada mês, sempre na última semana.

Identificação das espécies. Aidentificação dos exemplares foi feita de acordo com a classificação proposta por Young \& Duncan $^{38}$ e Martins cols ${ }^{26}$. Os espécimens com caracteres perdidos ou danificados que impossibilitaram a identificação a nível específico foram considerados Lutzomyia spp.

Reação de precipitina. Os flebotomíneos capturados foram transportados vivos até o laboratório, onde as fêmeas engurgitadas foram sacrificadas por congelamento para total paralisação do processo digestivo. Sete diferentes anti-soros foram utilizados neste estudo ( boi, cavalo, porco, roedor, cão, homem e galinha). 0 método e as técnicas usadas para preparar e identificar os insetos alimentados foram realizadas de acordo com Lorosa cols, em 199824.

\section{RESULTAD0S}

Durante 0 período de janeiro a dezembro de 2002, foram capturados 1.408 flebotomíneos no município de Porteirinha distribuídos em 14 espécies: Brumptomyia avellari (Costa Lima, 1932) , Lutzomyia capixaba Dias, Falcão, Silva \& Martins, 1987, L evandroi (Costa Lima, 1932), L intermedia (Lutz \& Neiva, 1912) , L lenti (Mangabeira, 1938) , L longipalpis ( Lutz \& Neiva, 1912), L peresi (Mangabeira, 1942), L quinquefer (Dyar, 1929) , L renei ( Martins, Falcão e Silva, 1957) , L sallesi ( Galvão \& Coutinho, 1939) , L sordellii (Shannon \& Del Ponte, 1927), L termitophila Martins, Falcão \& Silva, 1964, L trinidadensis (Newstead, 1922) e L whitmani (Antunes \& Coutinho, 1939). De acordo com 0 ambiente, os resultados obtidos mostram que 0 peridomicílio apresenta a maior porcentagem das espécies encontradas na região ( $53,3 \%$ ) , embora parte da fauna também tenha sido encontrada no intradomicílio (46,7\%), com uma maior proporção de machos em relação às fêmeas (Tabela 1).

A Tabela 2 apresenta 0 número mensal de flebotomíneos capturados com armadilha luminosa CDC, segundo bairros e sexo, no município de Porteirinha, no período de janeiro a 
Tabela 1 - Flebotomíneos capturados em armadilha tipo CDC segundo espécie, ambiente e sexo, no período de janeiro a dezembro de 2002, em Porteirinha, MG.

\begin{tabular}{|c|c|c|c|c|c|c|}
\hline \multirow[b]{2}{*}{ Espécies } & \multicolumn{2}{|c|}{ Intradomicílio } & \multicolumn{2}{|c|}{ Peridomicílio } & \multirow[b]{2}{*}{ Total } & \multirow[b]{2}{*}{ Porcentagem } \\
\hline & $\mathrm{M}$ & $F$ & M & $F$ & & \\
\hline Brumptomyia avellari & 1 & 3 & 1 & 5 & 10 & 0,7 \\
\hline Lutzomyia capixaba & 0 & 4 & 0 & 1 & 5 & 0,4 \\
\hline Lutzomyia evandroi & 0 & 0 & 1 & 0 & 1 & 0,1 \\
\hline Lutzomyia intermedia & 9 & 8 & 12 & 10 & 39 & 2,8 \\
\hline Lutzomyia lenti & 64 & 62 & 86 & 63 & 275 & 19,5 \\
\hline Lutzomyia longipalpis & 293 & 129 & 366 & 128 & 916 & 65,1 \\
\hline Lutzomyia peresi & 3 & 12 & 1 & 6 & 22 & 1,6 \\
\hline Lutzomyia quinquefer & 3 & 7 & 0 & 2 & 12 & 0,8 \\
\hline Lutzomyia renei & 0 & 0 & 0 & 1 & 1 & 0,1 \\
\hline Lutzomyia sallesi & 9 & 14 & 8 & 25 & 56 & 3,9 \\
\hline Lutzomyia sordellii & 1 & 7 & 4 & 2 & 14 & 1,0 \\
\hline Lutzomyia termitophila & 0 & 0 & 1 & 7 & 8 & 0,6 \\
\hline Lutzomyia trinidadensis & 11 & 1 & 2 & 2 & 16 & 1,1 \\
\hline Lutzomyia whitmani & 0 & 0 & 1 & 1 & 2 & 0,1 \\
\hline Lutzomyia spp & 2 & 14 & 4 & 11 & 31 & 2,2 \\
\hline Total & 396 & 261 & 487 & 264 & 1.408 & 100,0 \\
\hline
\end{tabular}

Tabela 2 - Número mensal de flebotomíneos capturados em armadilha tipo CDC, segundo local e sexo. Município de Porteirinha, MG, no período de janeiro a dezembro de 2002.

\begin{tabular}{|c|c|c|c|c|c|c|c|c|c|c|c|c|c|c|}
\hline \multirow[b]{2}{*}{ Mês } & \multicolumn{2}{|c|}{ Renascença } & \multicolumn{2}{|c|}{ São Judas } & \multicolumn{2}{|c|}{ São Sebastião } & \multicolumn{2}{|c|}{ Vila União } & \multicolumn{2}{|c|}{ Vila Serranópolis } & \multicolumn{2}{|c|}{ Vila Kennedy } & \multicolumn{2}{|c|}{ Vila Vitória } \\
\hline & $\bar{M}$ & $\bar{F}$ & M & $\bar{F}$ & M & F & M & $\bar{F}$ & M & $\mathrm{F}$ & M & F & $\overline{\mathrm{M}}$ & $\bar{F}$ \\
\hline jan & 1 & 1 & 46 & 37 & 3 & 1 & 6 & 10 & 14 & 7 & 0 & 1 & 6 & 2 \\
\hline fev & 7 & 6 & 46 & 24 & 2 & 1 & 9 & 11 & 19 & 12 & 0 & - & 5 & 7 \\
\hline $\operatorname{mar}$ & 4 & 8 & 75 & 38 & 1 & 0 & 22 & 10 & 8 & 8 & 0 & - & 12 & 8 \\
\hline$a b r$ & 3 & 4 & 25 & 15 & 9 & 12 & 15 & 3 & 6 & 3 & 1 & 2 & 4 & - \\
\hline mai & 3 & 8 & 29 & 12 & 4 & 4 & 11 & 8 & 8 & 12 & 3 & - & 8 & 3 \\
\hline jun & 5 & 6 & 18 & 8 & 1 & 0 & 3 & 2 & 13 & 7 & 1 & - & 3 & 2 \\
\hline jul & 21 & 7 & 11 & 5 & 3 & 0 & 4 & 6 & 4 & 4 & 2 & - & 5 & 5 \\
\hline ago & 36 & 9 & 14 & 6 & 4 & 5 & 12 & 7 & 5 & 3 & 2 & 3 & 3 & 3 \\
\hline set & 27 & 11 & 27 & 16 & 2 & 1 & 12 & 2 & 7 & 11 & 1 & 2 & 4 & 2 \\
\hline out & 4 & 8 & 82 & 29 & 2 & 4 & 9 & 7 & 1 & 3 & 0 & - & 2 & 2 \\
\hline nov & 17 & 3 & 60 & 24 & 1 & 1 & 7 & 11 & 1 & 1 & 4 & 7 & 5 & 7 \\
\hline dez & 13 & 1 & 4 & 0 & 3 & 0 & 0 & 1 & 0 & 0 & 0 & 1 & 3 & 4 \\
\hline Subtotal & 141 & 72 & 437 & 214 & 35 & 29 & 110 & 78 & 86 & 71 & 14 & 16 & 60 & 45 \\
\hline Total & \multicolumn{2}{|c|}{213} & \multicolumn{2}{|c|}{651} & \multicolumn{2}{|c|}{64} & \multicolumn{2}{|c|}{188} & \multicolumn{2}{|c|}{157} & \multicolumn{2}{|c|}{30} & \multicolumn{2}{|c|}{105} \\
\hline
\end{tabular}

dezembro de 2002. A ocorrência de flebotomíneos foi maior nos bairros São Judas ( 46,2\%), seguida dos bairros Renascença $(15,1 \%)$ e Vila União $(13,3 \%)$.

ATabela 3 apresenta os índices de fêmeas de L longipalpis segundo 0 teste de precipitina com utilização de diversos

Tabela 3 - Índices de fêmeas de Lutzomyia longipalpis segundo o teste de precipitina com utilização de diversos anti-soros, em Porteirinha, MG.

\begin{tabular}{lcc}
\hline Anti-soro & Número absoluto & Porcentagem relativa \\
\hline Boi & 4 & 10,5 \\
Cão & 5 & 13,2 \\
Cavalo & 10 & 26,3 \\
Galinha & 10 & 26,3 \\
Homem & 2 & 5,3 \\
Porco & 0 & 0 \\
Roedor & 6 & 15,8 \\
Năo reagente & 1 & 2,6 \\
\hline Total & 38 & 100,0 \\
\hline
\end{tabular}

anti-soros. Observa-se que esta espécie alimentou-se preferencialmente em galinhas e cavalos (26,3\%), mas também foi encontrada alimentada de sangue de roedores (15,8\%), cães $(13,2 \%)$, bois $(10,5 \%)$ e homem $(5,3 \%)$.

\section{DISCUSSÃ0}

A leishmaniose visceral era uma doença praticamente silvestre, característica de ambientes rurais, que tem sofrido uma mudança do perfil epidemiológico, fundamentalmente causada por modificações sócioambientais, como 0 desmatamento e 0 processo migratório de populações humana e canina originárias de áreas rurais onde a doença é endêmica. Além disso, 0 crescimento desordenado das cidades levando à destruição do meio ambiente, associado ao aumento da crise social, tem sido apontados como os principais fatores promotores das condições adequadas para ocorrência da LV na área urbana ${ }^{15}$. 
A mudança no contexto da doença, induzida pela adaptação de vetores à nova realidade é fato já bastante conhecido. A devastação de grandes áreas silvestres para exploração econômica traz a doença para a periferia dos centros urbanos, sendo que tanto os vetores como os hospedeiros são obrigados a migrarem para 0 peridomicílio humano em busca de alimentos, transmitindo ao mesmo tempo os agentes da doença ${ }^{31}$.

0 processo adaptativo de espécies vetoras encontrado em várias áreas urbanas também pôde ser observado no município de Porteirinha. Os bairros com o maior número de flebotomíneos capturados caracterizam-se por serem locais onde devastações foram praticadas tornando as matas escassas, formando ilhas de vegetações isoladas capões. As casas situam-se, de modo geral, na confluência dos declives de serras que formam os chamados boqueirões ou pés-de-serra. Estes fatores sócioambientais associados à baixa condição econômica dos moradores contribuem de forma marcante na transmissão da LV .

Houve uma nítida predominância de L longipalpis, tanto no ambiente intradomiciliar quanto no peridomicílio em relação às demais espécies ( Tabela 1). Aocorrência desta espécie dentro e nos arredores da casa demonstra que ela se encontra bastante adaptada aos mais diversos ambientes.

A presença de animais domésticos e silvestres no peridomicílio atrai um grande número de flebotomíneos, conseqüentemente, algumas espécies que são vetoras de leishmanioses, contribuem assim, para o aumento do risco de transmissão de Leishmania sp 511121316 .

0 estudo do conteúdo estomacal de insetos hematófagos é de grande importância ecológica e epidemiológica, pois além de permitir a identificação dos hospedeiros sobre os quais os flebotomíneos se alimentam, pode indicar os reservatórios potenciais de leishmânias. Os dados da literatura mostram que 0 caráter oportunista parece predominar na alimentação desses insetos que podem sugar ampla variedade de vertebrados ${ }^{28} 29$.

A busca por fontes de alimentação é uma resposta comportamental que afeta a reprodução e a densidade populacional das espécies. Fêmeas requerem sangue de vertebrados para a maturação de seus ovos. Assim, dependendo do seu grau de adaptação às condições ambientais modificadas pelo homem, algumas espécies podem ser mais facilmente encontradas em ambientes peridomiciliares que outras.

A atração de L longipalpis por humanos foi confirmada por Quinnell e cols ${ }^{29}$. Na Costa Rica, Zeledon e cols ${ }^{39}$ também capturaram números significativos de flebótomos em iscas humanas, mas também no cão, porco cavalo e boi ${ }^{39}$. Entretanto, Morrison e cols ${ }^{28}$ sugeriram que esta espécie é oportunista e na Colômbia, não é fortemente atraída por humanos e cães ${ }^{28}$. Aguiar cols ${ }^{1}$ também mostraram uma clara preferência de flebotomíneos por galinhas ${ }^{1}$.

A presença contínua de L longipalpis e a capacidade desta espécie em se alimentar em uma grande variedade de vertebrados são de bastante relevância epidemiológica, pois pode fornecer dados que irão subsidiar a escolha, pelos órgãos competentes, dos métodos de controle da doença mais adequados à situação atual.

\section{AGRADECIMENTOS}

Aos moradores do município de Porteirinha, pela simplicidade e colaboração e à Fundação Nacional de Saúde, pelo apoio técnico e logístico na execução deste trabalho.

\section{REFERÊNCIAS BIBLIOGRÁFICAS}

1. Aguiar GM, Vilela ML, Lima RB. Ecology of the sandflies of Itaguaí, an area of cutaneous leishmaniasis in the State of Rio de Janeiro. Food preferences (Diptera, Psychodidade, Phlebotominae). Memórias do Instituto Oswaldo Cruz 82: 583-584, 1987.

2. Aguilar CM, Fernandez E, Fernandez R, Cannova DC, Ferrer E, Cabrera E, Souza WJS, Coutinho SG. Urban visceral leishmaniasis in Venezuela. Memórias do Instituto Oswaldo Cruz 93: 15-16, 1998.

3. Barata RA, França-Silva JC, Fortes-Dias CL, Costa RT, Silva JC, Vieira EP, Prata A, Michalsky EM, Dias ES. Sand flies in Porteirinha, an endemic area of American Visceral Leishmaniasis in the State of Minas Gerais, Brazil. Memórias do Instituto Oswaldo Cruz 99:481-487, 2004.

4. Barros VL, Rebêlo JMM, Silva FS. Flebotomíneos (Diptera:Psychodidae) de capoeira do município de Paço do Lumiar, Estado do Maranhão, Brasil. Área de transmissão de leishmaniose. Cadernos de Saúde Pública 16: 265$270,2000$.

5. Brazil RP, De Almeida DC, Brazil BG, Mamede SMPO. Chicken house as a resting site of sandflies in Rio de Janeiro, Brazil. Parasitologia 33: 113117, 1991.

6. Deane LM, Deane MP. Encontro de leishmanias nas vísceras e na pele de uma raposa em zona endêmica de calazar, nos arredores de Sobral. 0 Hospital 45: 419-421, 1954.

7. Deane LM, Deane MP. Observações preliminares sobre a importância comparativa do homem, do cão e da raposa (Lycalopex vetulus) como reservatórios da Leishmania donovani, em área endêmica de calazar no Ceará. 0 Hospital 48: 61-76, 1955.

8. Deane LM, Deane MP. Leishmaniose visceral urbana (no cão e no homem) em Sobral, Ceará. 0 Hospital 47: 75-87, 1956.

9. Deane LM, Deane MP. Visceral leishmaniasis in Brazil. Geographical distribution and transmission. Revista do Instituto de Medicina Tropical 4: 149-212, 1962.

10. Dedet JP. Leishmania et leishmaniose du Continent américan. Annales de L'Institut Pasteur 4: 3-26, 1993.

11. Forattini OP. Nota sobre criadouros naturais de flebótomos em dependências peridomiciliares, no Estado de São Paulo. Arquivos da Faculdade de Higiene e Saúde Pública 7: 158-167, 1953.

12. Forattini OP. Sobre os reservatórios naturais da Leishmaniose Tegumentar Americana. Revista do Instistuto de Medicina Tropical 2: 195-200, 1960.

13. Forattini OP. Observações feitas sobre a transmissão da Leishmaniose Tegumentar no Estado de São Paulo, Brasil. Revista de Saúde Pública 10: 31-43, 1976.

14. França-Silva JC, Costa RT, Siqueira AM, Machado-Coelho GLL, Mayrink W, Vieira EP, Costa JC, Genaro 0, Nascimento E. Epidemiology of canine visceral leishmaniosis in the endemic area of Montes Claros municipality, Minas Gerais State, Brazil. Veterinary Parasitology 111:161-173, 2003.

15. Fundação Nacional de Saúde. Leishmaniose Visceral no Brasil: situação atual, principais aspectos epidemiológicos, clínicos e medidas de controle. Boletim eletrônico epidemiológico 6, 2002.

16. Gomes AC, Rabello EX, Santos JLF, Galati EAB. Aspectos epidemiológicos da Leishmaniose Tegumentar Americana. 3. Observações naturais sobre 0 ritmo diário de Psychodopigus intermedius em ambiente florestal e extraflorestal. Revista de Saúde Pública 17: 23-30, 1983.

17. Grimaldi Jr G, Tesh RB, Pratt DM. A review of geographical distribution and epidemiology of leishmaniasis in the New World. American Journal of Tropical Medicine and Hygiene 41: 687-725, 1989. 
18. Instituto Brasileiro de Geografia e Estatística. Anuário Estatístico do Brasil - Instituto Brasileiro de Geografia e Estatística, 1997.

19. Instituto Brasileiro de Geografia e Estatística. Anuário Estatístico do Brasil - Instituto Brasileiro de Geografia e Estatística, 2000.

20. Lainson R. Demographic changes and their influence on the epidemiology of the American leishmaniases. In: Service MW (ed) Demography and Vector-Borne Diseases. CRC Press, Boca Raton, p. 85-106, 1989.

21. Lainson R, Shaw JJ, Ryan L, Ribeiro RS, Silveira FT. Leishmaniasis in Brazil. XXI. Visceral leishmaniasis in the Amazon Region and further observations on the role of Lutzomyia longipalpis ( Lutz \& Neiva, 1912) as the vector. Transactions of the Royal Society of Tropical Medicine and Hygiene 79: 223-226, 1985.

22. Lainson R, Shaw JJ, Ryan L, Silveira FT, Fraiha H. Leishmaniasis in Brazil. XIX: Visceral leishmaniasis in the Amazon region, and the presence of L. longipalpis on the island of Marajó, Para State. Transactions of the Royal Society of Tropical Medicine and Hygiene 77: 323-330, 1983.

23. Lainson R, Shaw JJ, Silveira FT, Braga RR. American visceral leishmaniasis: on the origen of Leishmania (Leishmania) chagasi. Transactions of the Royal Society of Tropical Medicine and Hygiene 81: 517, 1987.

24. Lorosa ES, Andrade RE. Identificação de fontes alimentares de mosquitos no município de Nova Iguaçú, RJ, Brasil, pela técnica da reação de precipitina. Entomology y Vector 5: 85-92, 1998.

25. Luz ZMP, Pimenta DN, Cabral ALLV, Fiúza VO, Rabello AL. A urbanização das leishmanioses e a baixa resolutividade diagnóstica em municípios da Região Metropolitana de Belo Horizonte. Revista da Sociedade Brasileira de Medicina Tropical 34: 249-254, 2001

26. Martins AVP, Williams P, Falcão AL. American sand flies (Diptera, Psychodidae, Phlebotominae). Academia Brasileira de Ciências, Rio de Janeiro, Brazil, 1978.

27. Marzochi MCA. A leishmaniose tegumentar no Brasil. In: Grandes Endemias Brasileiras. Editora Universidade de Brasília, Brasilia, 1989.

28. Morrison AC, Ferro C, Tesh RB. Host preferences of the sandfly at an endemic focus of American Visceral Leishmaniasis in Colombia. American Journal of Tropical Medicine and Hygiene 49: 68-75, 1993.

29. Quinnell RJ, Dye C, Shaw JJ. Host preferences of the sandfly Lutzomyia longipalpis in Amazonian Brazil. Medicine and Veterinary Entomology 6: 195-200, 1992.
30. Rebêlo JMM, Leonardo FS, Costa JM, Pereira YN, Silva FS. Sandflies (Diptera. Psychodidae) from an endemic leishmaniasis area in the cerrado region of the State of Maranhão, Brazil. Cadernos de Saúde Pública 15: 623-630, 1999

31. Rosa ICAS. Perfil epidemiológico dos casos de leishmaniose tegumentar americana notificados na Região Metropolitana de Belo Horizonte Minas Gerais, 1989 a 1995. Dissertação de Mestrado. Universidade Federal de Minas Gerais, Belo Horizonte, MG, 1997.

32. Rosabal R, Trejos A. Phlebotomus de El Salvador ( Diptera, Psychodidae). II- Observaciones sobre su biologia con especial referencia a P. longipalpis. Revista de Biologia Tropical 13: 219-228, 1965.

33. Sherlock IA, Miranda JC, Sadigursky M, Grimaldi Junior G. Natural infection of the opossum Didelphis albiventris (Marsupialia, Didelphidae) with Leishmania donovani, in Brazil. Memórias do Instituto Oswaldo Cruz 79: 511, 1984.

34. Silva ES, Gontijo CMF, Pacheco RS, Fiuza VOP, Brazil RP. Visceral Leishmaniasis in the Metropolitan region of Belo Horizonte, State of Minas Gerais, Brazil. Memórias do Instituto Oswaldo Cruz 96: 285-291, 2001.

35. Sudia WA, Chamberlain RW. Battery-Operated light trap: an improved model. Mosquito News 22: 126-129, 1962.

36. Travi BL, Adler GH, Lozano M, Cadena H, Montoya-Lerna J. Impact of habitat degradation on phlebotominae (Diptera: Psychodidae) of tropical dry forests in Northern Colombia. Journal of Medical Entomology 39: 451456, 2002.

37. Ximenes MFFM, Souza MF, Castellón EG. Density of sandflies (Diptera: Psychodidae) in domestic and wild animal shelters in an area of visceral leishmaniasis in the State of Rio Grande do Norte, Brazil. Memórias do Instituto Oswaldo Cruz 94: 427-432, 1999.

38. Young DG, Duncan MA. Guide to the identification and geographic distribution of Lutzomyia sand flies in Mexico, the West Indies, Central and South America (Diptera: Psychodidae). Memoirs of the American Entomological Institute 54: 1-881, 1994

39. Zeledon R, Murrillo J, Gutierrez H. Observaciones sobre la ecologia de Lutzomyia longipalpis ( Lutz \& Neiva, 1912) y posibilidades de existencia de leishmaniasis visceral en Costa Rica. Memórias do Instituto Oswaldo Cruz 79: 455-459, 1984 